\title{
Depth Estimation using FCNN
}

\author{
Pallavi Parlewar ${ }^{*}$, Siddhant Kumar ${ }^{2}$, Bhumika Neole ${ }^{3}$ and Manoj Sarve ${ }^{4}$ \\ ${ }^{134}$ Shri Ramdeobaba College of Engineering and Management, Nagpur, India \\ ${ }^{2}$ Center Point School Wardhman Nagar, Nagpur, India
}

\section{ABSTRACT}

This paper presents a novel supervised structure for estimating the depth from the monocular images. Floating point Convolution Neural Network (FCNN) which shows better results as compared to traditional algorithms. Rectified Linear Unit (ReLU) is the used as an activation function in deep learning models. As ReLu shows performance improvement between the layers as the data transferred is compatible to the ranges prescribe by the layers. KITTI and City Space dataset is use for training and testing. We have use most commonly dataset so that the benchmarking is done on the basis of same dataset and same parameter, Root Mean Square Error(RMSE) and Del< $1.25 * 3$. Our purposed FCNN algorithm yields improved results in depth estimation of various images.

\section{KEY WORDS: DEEP LEARNING, FCNN, DEPTH ESTIMATION, RECTI LINEAR UNIT.}

\section{INTRODUCTION}

A fundamental challenge in computer vision is to estimate the depth of scene from images. Industrial applications such as self-driving platforms, robotics, augmented reality and localization and navigation systems are the areas where estimating depth from images and video is crucial. Object detection specifically for medical images and video reconnaissance which, when it comes to practical applications cannot target three-dimensional positions. Thus, it is important for practical purposes to combine information off depth to obtain three-dimensional object detection. Depth estimation is done through supervised and unsupervised learning network.

Anjie Wang et. al. perceptual loss for depth estimation is done using unsupervised learning framework. From the video sequence the depth and camera motion is estimated

\section{ARTICLE INFORMATION}

*Corresponding Author: parlewarpk@rknec.edu

Received 20th Oct 2020 Accepted after revision 21st Dec 2020

Print ISSN: 0974-6455 Online ISSN: 2321-4007 CODEN: BBRCBA

Thomson Reuters ISI Web of Science Clarivate Analytics USA and Crossref Indexed Journal

\section{Clarivate
Analytics}

NAAS Journal Score 2020 (4.31)

A Society of Science and Nature Publication,

Bhopal India 2020. All rights reserved.

Online Contents Available at: http//www.bbrc.in/

Doi: http://dx.doi.org/10.21786/bbrc/13.14/86 by training the network. The network gives depth and position of the scene which can be used for reconstructing the scene. The quality of the scene is assess by extracting features from the pretrained network and the experimental results shows improved estimation accuracy. Delong Yang et. al showed improved smoothness by using adaptive loss function. Yang et.al used successive stereo images to training of the model and only one image was used for testing. Eigen et al. used two layer to train depth network by using their corresponding ground truth. Huoling Luo et.al. estimated depth for laparoscopy images using unsupervised learning by training an encoder and decoder of convolutional neural networks model. Loss function is calculated and smoothness is done to get smooth depth surface.

The result shows that the depth of the proposed site is nearer to the real surgical site and reserves all the truthful details of the site. Junning Zhang et al. projected the UnLearner visual odometer, a novel unsupervised deep learning pipeline. To confine the use of the network, the system uses geometric consistency loss of temporal images. Unlearner visual odometry can also estimate camera motion between inconsecutive frames which allows UnLearner visual odometry to be well appropriate in scenes with large camera motion. Lipu Zhou and Michael Kaess evaded over fitting using windowed bundle adjustment framework to train the network. Context

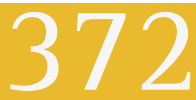


information attenuation had been eliminated using spatial pyramid net and super resolution net is castoff to recover details of the image which are the modifications in the U Net architecture. Clip loss function was proposed for designing complicated network to grip the moving object and occlusions. Shuhan Chen et. al. Salient object detection using full convolution based deep learning.

To guide feature learning in side output layers they first introduced a visual attention mechanism, attention weight is employed in a top-down manner to bridge high level logic information and subsequently filtering technique is used to remove background noise. Xiao Wang et. al. Saliency is detected from an encoder decoder framework using Deep Reinforcement Learning. In this the author has taken the adaptive weight of each domain so as to take the decision and is trained through discrete action selection with content loss and adversarial loss to interact with the environment. Depth and ego motion estimation is demonstrated using unsupervised stacked reproductive adversarial network. Lowermost layer of stack helps in estimating depth and ego motion where as higher layers are used in estimating spatial features.

Layers are recurrently represented to capture the temporal data. To evade the fault aliasing of multiple camera pose. Smoothing of uneven and unsmooth scene is done by using upsampling module in the encoder-decoder of the network. Xiaojiao Tang and lifang Then uses two aspect for learning unsupervised monocular image depth prediction. In first aspect they used multi-scale feature extraction for monocular image depth estimation the second part is deconvolution prediction network. In the second aspect they trained the model with asymmetric parameter. Image reconstruction is done by combining MS-SSIM algorithm and L1 algorithm. Images with mirrors, transparent, and shadows shows good Prediction accuracy.

\section{METHODOLOGY}

In this section first we will deal with motivation of this work, overview and network architecture. We will introduce depth estimation network with how the network is train to get accuracy and RSME. With the wide application of depth estimation researchers are focussing on augmented reality. In coming days augmented reality may be our daily routine gadget. This may replace our today's very important gadgets such as mobile, laptops etc. Augmented reality in medical imaging will play vital role in days to come because it makes the anatomy of intent visible part to surgeon which otherwise is not visible. It results in better surgical precision and therefore potentially better surgical outcomes and faster recovery times.

For example by stacking $2 \mathrm{D}$ images we get $3 \mathrm{D}$ view of the object. 2D MRI scan images and calculating the depth estimation of the organ which on superimposed on the patient body using augmented reality can help to pin point the location of injuries and tumour and help the surgeon during surgery. So augmented reality is reliant on depth estimation. Depth estimation using deep learning gives better accuracy. In this work we have used Floating Convolution Neural Network (FCNN) for depth estimation. Floating CNN does not have a fully connected output layer.Floating point numbers are castoff in lieu of the weights. The Rectified Linear Unit(ReLU) is the usually used as an activation function in deep learning models. The function receives any negative input it returns 0 , but returns the same value back for any positive value. Following is the steps of the algorithm:

Step1. Collect input images and ground truth images

Step 2. Train a CNN that can take an input image and produce an output image

Step 3. The following architecture is used for the floating CNN,

a. Initialize a sequential $\mathrm{CNN}$

b. Add a Conv2D layer of size 32×32 with window size of $3 \times 3$, a linear activation function and similar padding

c. Add a leaky ReLU with alpha=0.1

d. Add a Conv2D layer of size $64 \times 64$ with window size of $3 \times 3$, a linear activation function and similar padding e. Add a leaky ReLU with alpha=0.1

f. Add a Max Pooling layer of size $2 \times 2$

g. Add a Conv2D layer of size $128 \times 128$ with window size of $3 \times 3$, a linear activation function and similar padding

h. Add a leaky ReLU with alpha=0.1

i. Add a Max Pooling layer of size $2 \times 2$

j. Add a $1 \times 1$ Conv2D layer of window size $3 \times 3$, producing the final output

Step 4. Use a sparse categorical cross entropy based learning mechanism

Step 5. Train the network with different images, to get the final trained CNN

Step 6. Give a new image to the CNN to obtain the final depth map

Figure 1: Proposed depth estimation architecture of FCNN structure based on ReLu

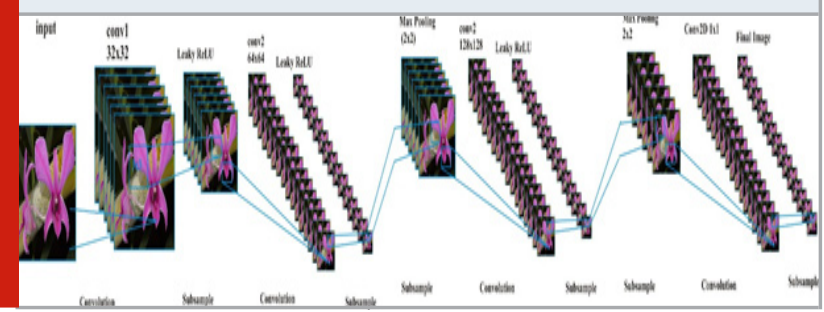

\section{RESULTS AND DISCUSSION}

This section deals with the comparison with the stateof -art algorithms. Network is implemented using Tensorflow 1.9 and is trained. KITTI and City space dataset is used for training set and test set. 9325 images for testing and 6528 images for testing out of which 1398 for validation. For evaluation of the FCNN architecture 3 different methods are used to compare the proposed work. To carry out the trials and evaluation for all algorithm same crop is used in all input images. 
Figure 2: Depth estimation using FCNN for various images

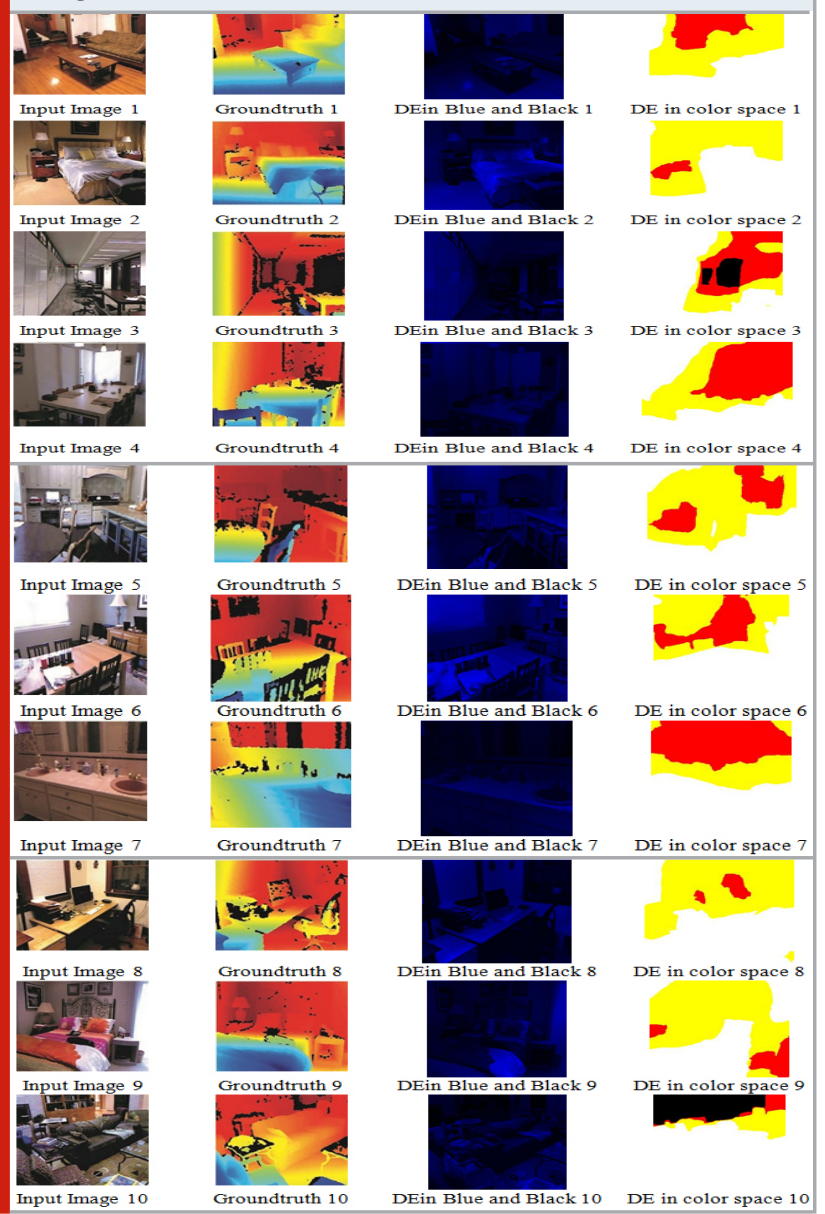

The size of the input image to the network is $256 \mathrm{X}$ 256, 256 X 256 for training and 256 X 256 in a depth map. The benchmarking is done on the basis of absolute relative difference (Abs Rel), Square related difference (Sq Rel), Root Mean Square Error(RMSE)and Log Root Mean Square Error(RMSE Log 10). Our algorithm perform better in comparison with the supervised and unsupervised algorithms because our architecture use Recti Linear unit due to which their performance improvement between the layers as the data transferred is compatible to the ranges prescribe by the layers.

Fig. (2) Show home interior data as input image and ground truth is already available with the KITTI dataset. Image 1 to 10 shows input images, ground truth images, depth estimated images, blue color indicate nearer and black far distance, similarly same depth estimated image is shown in red-yellow-white-black color space in which white shows to be nearer and black indicates to be far point Table: 1shows the results of various image extracted from KITTI dataset with ground truth. Our algorithm outperform for Del $<1.25^{\wedge} 3$. Table: 2 shows the results of three different algorithms. The table is divided into several parts according to the supervised and unsupervised algorithms. Our algorithm shows better result for Del $<1.25^{*} 3$ and RMSE. Our algorithms learn from scrape without demanding more information and outperforms.

Fig.2 KITTI dataset is used to show qualitative results, Right to left FCNN output white-red-yellow-black colour space, our result in blue-black, ground truth from KITTI dataset and input images

Table 1. Results of various images. Ground truth is use from KITTI dataset

\begin{tabular}{|c|c|c|c|c|c|c|c|c|}
\hline \multirow[b]{2}{*}{ Image } & \multirow[b]{2}{*}{ Size } & \multicolumn{4}{|c|}{ Lower is better } & \multicolumn{3}{|c|}{ Higher is better } \\
\hline & & $\begin{array}{l}\text { Absolute } \\
\text { Relative } \\
\text { difference }\end{array}$ & $\begin{array}{c}\text { Squared } \\
\text { Relative } \\
\text { difference }\end{array}$ & $\begin{array}{l}\text { Root } \\
\text { Mean } \\
\text { Square } \\
\text { Error }\end{array}$ & $\begin{array}{c}\text { Root } \\
\text { Mean } \\
\text { Square } \\
\text { Error Log10 }\end{array}$ & $\begin{array}{c}\text { Del } \\
<1.25\end{array}$ & $\begin{array}{c}\text { Del< } \\
1.25^{\wedge} 2\end{array}$ & $\begin{array}{c}\text { Del< } \\
1.25^{\wedge} 3\end{array}$ \\
\hline 1 & $256 * 256$ & 0.8624 & 1.6884 & 2.5576 & 0.4078 & 0.8916 & 0.9312 & 0.9906 \\
\hline 2 & $256 * 256$ & 0.6064 & 1.6061 & 1.1879 & 0.07478 & 0.83539 & 0.8725 & 0.9282 \\
\hline 3 & $256 * 256$ & 0.7366 & 1.6538 & 2.2964 & 0.3610 & 0.8946 & 0.9344 & 0.9940 \\
\hline 4 & $256 * 256$ & 0.7623 & 1.4522 & 3.004 & 0.4777 & 0.8979 & 0.9378 & 0.9977 \\
\hline 5 & $256 * 256$ & 0.6820 & 1.6501 & 1.9809 & 0.2968 & 0.8873 & 0.9267 & 0.9859 \\
\hline 6 & $256 * 256$ & 0.8494 & 0.8560 & 0.86072 & -0.0651 & 0.8051 & 0.8409 & 0.8945 \\
\hline 7 & $256 * 256$ & 0.51825 & 0.9795 & 4.170 & 0.6201 & 0.8982 & 0.9381 & 0.9980 \\
\hline 8 & $256 * 256$ & 0.7056 & 1.3383 & 1.7537 & 0.2439 & 0.8715 & 0.9102 & 0.9683 \\
\hline 9 & $256 * 256$ & 0.7928 & 1.5169 & 1.8958 & 0.2778 & 0.8681 & 0.9066 & 0.9645 \\
\hline 10 & $256 * 256$ & 0.5496 & 1.3444 & 3.0349 & 0.4821 & 0.8906 & 0.9302 & 0.9895 \\
\hline
\end{tabular}


Table 2. KITTI and City space dataset is use for training set and test set. Experimental data of other method is taken from the paper which is given in the reference

\begin{tabular}{|l|c|c|c|c|c|c|c|c|}
\hline $\begin{array}{l}\text { Image } \\
\text { (.jpg) }\end{array}$ & Supervised & $\begin{array}{c}\text { Absolute } \\
\text { Relative } \\
\text { difference }\end{array}$ & $\begin{array}{c}\text { Squared } \\
\text { Relative } \\
\text { difference }\end{array}$ & $\begin{array}{c}\text { Root } \\
\text { Mean } \\
\text { Square } \\
\text { Error }\end{array}$ & $\begin{array}{c}\text { Root Mean } \\
\text { Square } \\
\text { Error } \\
\text { Log10 }\end{array}$ & Del < 1.25 & Del< $1.25^{\wedge} 2$ & Del< $1.25^{\wedge} 3$ \\
\hline Zhang[1] & YES & 0.144 & 1.391 & 5.869 & 0.241 & 0.803 & 0.928 & 0.969 \\
\hline Liu[4] & YES & 0.202 & 1.614 & 6.523 & 0.275 & 0.678 & 0.895 & 0.964 \\
\hline Godard [2] & NO & 0.140 & 0.976 & 0.471 & 0.232 & 0.818 & 0.931 & 0.969 \\
\hline Eigen [3] & NO & 0.214 & 1.605 & 6.563 & 0.292 & 0.673 & 0.884 & 0.957 \\
\hline Ours & Yes & 0.518 & 0.979 & 4.17 & 0.620 & 0.898 & 0.938 & 0.998 \\
\hline
\end{tabular}

\section{CONCLUSION}

In this paper we have propose a novel FCNN network for depth estimation in monocular images. Because of Recti linear unit the data is transferrable to the prescribe ranges which increases the performance of the algorithm. In comparison with the existing supervised learning network our FCNN can generate more accurate result. In future we will extend our work with unsupervised learning method and also increase data set for training and testing.

\section{REFERENCES}

A P Nilawar, C G Dheete. Performance Evaluation of Content-Based Image Retrieval Using Convolutional Neural Network. International Journal of Future Generation Communication and Networking, Vol 13, No 3, May.2020.

Anjie Wang, Zhijun Fang1, Yongbin Gao, Xiaoyan Jiang, Siwei Ma. Depth Estimation of Video Sequences with Perceptual Losses. IEEE access, vol:6, p. 3053630546, June. 2018

C. Godard, 0. Mac Aodha, and G. J. Brostow, "Unsupervised monocular depth estimation with left-right consistency," Sep. 2016, arXiv:1609.03677, [Online]. Available: https://arxiv.org/abs/1609.03677

D. Eigen, C. Puhrsch, and R. Fergus, "Depth map prediction from a single image using a multi-scale deep network," in Proc. NIPS, 2014, p. 2366_2374.

Delong Yang, Xunyu Zhong, Lixiong Lin, Xiafu Peng. An Adaptive Unsupervised Learning Framework for Monocular Depth Estimation. IEEE access, vol:7, p.148142-148151,0ct. 2019.

Ehab H. El-Shazly, Xiaoyan Zhang, Jianmin Jiang. Improved appearance loss for deep estimation of image depth.Electronics Letters, vol. 55 no. 5, p. 264-266, Mar. 2019.

F. Liu, C. Shen, G. Lin, and I. Reid, “Learning depth from single monocular images using deep convolutional neural fields," IEEE Trans. Pattern Anal. Mach. Intell., vol. 38, no. 10, pp. 2024_2039, Oct. 2019.

H. Zhan, R. Garg, C. S. Weerasekera, K. Li, H. Agarwal, and I. Reid, "Unsupervised learning of monocular depth estimation and visual odometry with deep feature reconstruction," in Proc. CVPR, Jun. 2018,p. 340_349.

Huoling Luo, Qingmao Hu, Fucang Jia. Details preserved unsupervised depth estimation by fusing traditional stereo knowledge from laparoscopic images. Healthcare Technology Letters, vol: 6, Iss. 6, p. 154-158, 2019.

Junning Zhang, Qunxing Su, Pengyuan Liu, Chao Xu, Yanlong Chen. Unsupervised Learning of Monocular Depth and Large-Ego-Motion With Multiple Loop Consistency Losses. IEEE access, vol.7, p.77839-77848, June 2019.

Lipu Zhou, Michael Kaess. Windowed Bundle Adjustment Framework for Unsupervised Learning of Monocular Depth Estimation With U-Net Extension and Clip Loss. Ieee Robotics and Automation Letters, vol. 5, no. 2, p. 3283-3290 Apr. 2020.

Sakshi Wasnik, Pallavi Parlewar, Prashant Nimbalkar. Nodule detection in lung using multi-threshold segmentation, International Journal of Innovative Technology and Exploring Engineering (IJITEE), ISSN:2278-3075, vol. 8, Issue. 9, July 2019.

Sakshi Wasnik, Pallavi Parlewar, Prashant Nimbalkar, Detection of Cancerous Nodule in Lung Using KNN Classifier, Helix Vol. 9 (6): 5779- 5783, DOI 10.29042/2019-5779-5783.

Shuhan Chen, Ben Wang, Xiuli Tan, Xuelong Hu. IEEE Transactions on Cybernetics, vol: 50, Iss.5 , p. 20502062, May 2020.

Xiao Wang, Tao Sun, Rui Yang, Chenglong Li, Bin Luo, Jin Tang. Quality-Aware Dual-Modal Saliency Detection via Deep Reinforcement Learning. Signal Processing: Image Communication, vol.75, p. 158-167, July 2019. Xiaojiao Tang, Lifang Chen.An Unsupervised Monocular Image Depth Prediction Algorithm Based on Multiple Loss Deep Learning. IEEE access, vol:7, p.162405162414, Nov. 2019.

Wang, Y., Xu, Y. Unsupervised learning of accurate camera pose and depth from video sequences with Kalman filter. IEEE Access 2019, 7, 32796-32804. 